\title{
SOME PROBABILISTIC THEOREMS ON DIOPHANTINE APPROXIMATIONS $\left({ }^{1}\right)$ \\ BY

\author{
HARRY KESTEN
}

1. Introduction. Let

$$
\langle\xi\rangle=\min _{\boldsymbol{k} \text { integer }}|\xi-k|
$$

be the positive distance between $\xi$ and the nearest integer to $\xi$. The first theorem is concerned with

$$
\min _{1 \leqq k \leqq m}\langle k \xi\rangle .
$$

By the methods used for Theorem 1 we also solve some special cases of a problem raised in [2] concerning the existence of an integer $k$ for which $m \leqq k \leqq m c(c>1)$ and $\langle k \xi\rangle \leqq \alpha / k$ (Theorem 2 ).

While studying $\min _{1 \leq k \leq n}\langle k \xi\rangle$, one is naturally led to consider the number of integers $k$ for which $1 \leqq k \leqq m$ and $\langle k \xi\rangle \leqq \gamma$. The third theorem deals with this quantity in $p$ dimensions i.e. it considers $N(m, \gamma, p)$, the number of integers $k$, for which $1 \leqq k \leqq m$ and simultaneously

$$
\left\langle k \xi_{1}\right\rangle \leqq \gamma,\left\langle k \xi_{2}\right\rangle \leqq \gamma, \cdots,\left\langle k \xi_{p}\right\rangle \leqq \gamma
$$

Theorem 4 gives some easy generalizations of the third theorem.

Our approach is probabilistic in the sense that we do not take $\xi, \xi_{1}, \cdots, \xi_{p}$ fixed, but choose them randomly, according to a uniform distribution on $[0,1]$. This makes $\min _{1 \leq k \leq m}\langle k \xi\rangle$ and $N(m, \gamma, p)$ random variables. Accordingly, Theorem 1 gives an asymptotic expression for the Lebesgue measure of the set

$$
\left\{\xi: 0 \leqq \xi \leqq 1, m \cdot \min _{1 \leqq k \leqq m}\langle k \xi\rangle \leqq \alpha\right\} .
$$

Theorem 3, which states that $N(m, \gamma, p)$ has asymptotically $\left(p \rightarrow \infty, m \cdot(2 \gamma)^{p} \rightarrow \lambda\right)$ a Poisson distribution with mean $\lambda$, can be paraphrased similarly. It then gives asymptotic expressions for the $p$ dimensional Lebesgue measure of the sets

$$
\left\{\xi_{1}, \cdots, \xi_{p}: 0 \leqq \xi_{j} \leqq p, N(m, \gamma, p)=k\right\}, \quad k=0,1, \cdots
$$

Presented to the Society, May 22, 1961; received by the editors July 3, 1961.

(l) The research reported in this document has been sponsored in part by the Air Force Office of Scientific Research of the Air Research and Development Command, United States Air Force, through its European Office Contract no. AF 61(052)-187. 
We shall stick further to the probabilistic language. As far as used here it is of such a simple kind that it should not cause any difficulties. All the required definitions can be found in [9].

Theorems 1, 2 are immediate extensions of the results of Friedman and Niven [3] and of Erdös, Szüsz, and Turan [2]. Theorem 3 is proved by the method of moments. We are forced, however, to prove the convergence of the moments in a rather roundabout way (cf. also the remarks after Lemma 1). One should compare Theorem 3 with the well-known result of Dirichlet, which states that if $\gamma^{-1}$ is an integer, then there exists for every $\left(\xi_{1}, \cdots, \xi_{p}\right)$ at least one $k \leqq \gamma^{-p}$ satisfying (1.1). Our results show that the Lebesgue measure of the set of $\left(\xi_{1}, \cdots, \xi_{p}\right)$ 's, for which there exists such a $k \leqq \lambda(2 \gamma)^{-p}$, is approximately $1-e^{-\lambda}$. Related results were also obtained by Erdös and Rényi [1] who studied

$$
\max _{1 \leqq k \leqq m}\left|\sum_{j=1}^{p} e^{2 \pi i k \xi_{j}}\right| .
$$

2. The minimum of $\langle k x\rangle, k=1, \cdots, m$. Let $x$ have a uniform distribu$\operatorname{tion}\left({ }^{2}\right)$ on $[0,1]$ and put

$$
Z(m)=\min _{1 \leqq k \leqq m}\langle k x\rangle
$$

$$
N(m, \gamma)=\text { number of integers } k \text { for which } 1 \leqq k \leqq m \text { and }\langle k x\rangle \leqq \gamma,
$$

and

$$
t(\xi, \gamma)=\text { smallest integer } k \geqq 1 \text { for which }\langle k \xi\rangle \leqq \gamma .
$$

Clearly

is equivalent to

$$
Z(m) \leqq \alpha / m
$$

$$
N(m, \alpha / m) \geqq 1
$$

which in turn is equivalent to

$$
t(x, \alpha / m) \leqq m .
$$

Hence, if the limits exist $\left({ }^{3}\right)$,

$$
\begin{aligned}
\lim _{m \rightarrow \infty} P\{Z(m) \leqq \alpha / m\} & =\lim _{m \rightarrow \infty} P\{N(m, \alpha / m) \geqq 1\} \\
& =\lim _{m \rightarrow \infty} P\{t(x, \alpha / m) \leqq m\} .
\end{aligned}
$$

(2) We shall always use $x$ or $x_{i}$ for random variables, whereas fixed numbers from $[0,1]$ will be denoted by $\xi$ or $\xi_{i}$.

( $\left.{ }^{3}\right) P\{A\}=$ probability of the event $A, P\{A \mid B\}=$ conditional probability of the event $A$, given $B, E(X)=$ expectation of the random variable $X, E(X \mid B)=$ conditional expectation of the random variable $X$ given $B, \operatorname{var}(X)=E\left(X^{2}\right)-(E X)^{2}=$ variance of the random variable $X$, $\operatorname{var}(X \mid B)=E\left(X^{2} \mid B\right)-(E(X \mid B))^{2}=$ conditional variance of the random variable $X$ given $B$. 
The last limit in (2.6) can be found by the methods of Friedman and Niven [3] and of Erdös, Szüsz and Turan [2]. In [3] the first two moments of $t(x, \alpha / m)$ were computed. Let $F_{k}$ denote the Farey series of order $k$, that is the series of rational numbers $\left({ }^{4}\right) a / b, 0 \leqq a \leqq b \leqq k,(a, b)=1$, in ascending order (cf. [5, Chapter III for more details]). It was proved in [3, p. 27] that for every $\xi$

$$
t(\xi, 1 / n) \leqq n-1 .
$$

Writing further, for some fixed $n$,

$$
I\left(\frac{a}{b}\right)=\left[\frac{a}{b}-\frac{1}{n b}, \frac{a}{b}+\frac{1}{n b}\right]
$$

it was also shown in $[3$, p. 27] that

$$
t(\xi, 1 / n) \leqq k
$$

if and only if one has

$$
\xi \in I\left(\frac{a}{b}\right) \quad \text { for some } \frac{a}{b} \in F_{k}
$$

Moreover, there are at most two fractions $a / b \in F_{k}(k \leqq n-1)$ for which $\xi \in I(a / b)[2 ; 3]$. Let

$S_{k}=\left\{\xi: 0 \leqq \xi \leqq 1 ;\right.$ there exist $\frac{a_{1}}{b_{1}}, \frac{a_{2}}{b_{2}} \in F_{k}, \frac{a_{1}}{b_{1}} \neq \frac{a_{2}}{b_{2}}$

$$
\text { such that } \left.\xi \in I\left(\frac{a_{1}}{b_{1}}\right) \text { and } \xi \in I\left(\frac{a_{2}}{b_{2}}\right)\right\} \text {. }
$$

Thus $S_{k}$ is the subset of $[0,1]$ covered by two intervals $I$ from $F_{k}$. If $\mu\{\cdot\}$ denotes Lebesgue measure, then it follows from the above remarks that (cf. $[2$, formula $(12)]$ )

$$
\begin{aligned}
P\left\{t\left(x, \frac{1}{n}\right) \leqq k\right\} & =\sum_{a / b \in F_{k}} \mu\left\{I\left(\frac{a}{b}\right)\right\}-\mu\left\{S_{k}\right\}=\sum_{a / b \in F_{k}} \frac{2}{n b}-\mu\left\{S_{k}\right\} \\
& =\frac{2}{n} \sum_{b=1}^{k} \frac{\Phi(b)}{b}-\mu\left\{S_{k}\right\}
\end{aligned}
$$

where $\Phi(\cdot)$ is Euler's function [5, p. 52].

It remains to find an expression for $\mu\left\{S_{k}\right\}$. If $\xi$ is contained in two intervals $I\left(a_{1} / b_{1}\right), I\left(a_{2} / b_{2}\right)$ with $a_{i} / b_{i} \in F_{k}, k \leqq n-1$, then $\left[3\right.$, p. 27] $a_{1} / b_{1}$ and $a_{2} / b_{2}$ must be consecutive elements of $F_{k}$. It is known $[5$, Theorem 28, p. 23] that in this case

( $\left.{ }^{4}\right)[a]=$ largest integer which does not exceed $a$. Although we also use square brackets with other meaning, confusion seems unlikely. $(a, b)=$ greatest common divisor of $a$ and $b$. 


$$
\left|\frac{a_{1}}{b_{1}}-\frac{a_{2}}{b_{2}}\right|=\frac{\left|a_{1} b_{2}-a_{2} b_{1}\right|}{b_{1} b_{2}}=\frac{1}{b_{1} b_{2}}
$$

and hence

$$
\mu\left\{I\left(\frac{a_{1}}{b_{1}}\right) \cap I\left(\frac{a_{2}}{b_{2}}\right)\right\}=\left\{\begin{array}{cl}
\frac{b_{1}+b_{2}-n}{n b_{1} b_{2}} & \text { if } b_{1}+b_{2} \geqq n, \\
0 & \text { if } b_{1}+b_{2}<n .
\end{array}\right.
$$

One has therefore

$$
\mu\left\{S_{k}\right\}=\sum_{k}^{\prime} \frac{b_{1}+b_{2}-n}{n b_{1} b_{2}}
$$

where $\sum_{k}^{\prime}$ ranges over all pairs $a_{1} / b_{1}, a_{2} / b_{2}$ which are consecutive elements of $F_{k}$ and satisfy $b_{1}<b_{2}$ and $b_{1}+b_{2} \geqq n$. $\left(b_{1}=b_{2}\right.$ is impossible for successive fractions by Theorem 28, p. 23 in [5] and since we want to take every pair into account only once we may take $b_{1}<b_{2}$.) It was proved in Lemma 1 of [3] that for fixed $b_{1}<b_{2} \leqq k,\left(b_{1}, b_{2}\right)=1, b_{1}+b_{2} \geqq n$ there are exactly two choices for $a_{1}, a_{2}$ such that $a_{1} / b_{1}, a_{2} / b_{2}$ belong to $\sum_{k}^{\prime}$ while for $\left(b_{1}, b_{2}\right)>1$ there are no choices possible for $a_{1}, a_{2}$ (again by Theorem 28, p. 23 of [5]). Consequently

$$
\mu\left\{S_{k}\right\}=\sum_{k}^{\prime} \frac{b_{1}+b_{2}-n}{n b_{1} b_{2}}=\frac{2}{n} \sum_{b_{1}=1}^{k} \sum_{\substack{b_{2}=\max \left(n-b_{1}, b_{1}+1\right) \\\left(b_{1}, b_{2}\right)=1}}^{k} \frac{b_{1}+b_{2}-n}{b_{1} b_{2}} .
$$

With these preliminaries it is easy to prove

THEOREM 1.

$$
\begin{aligned}
\lim _{m \rightarrow \infty} P\left\{m \cdot \min _{1 \leqq k \leqq m}\langle k x\rangle \leqq \alpha\right\} & =\lim _{m \rightarrow \infty} P\left\{N\left(m, \frac{\alpha}{m}\right) \geqq 1\right\} \\
& =\lim _{m \rightarrow \infty} P\left\{t\left(x, \frac{\alpha}{m}\right) \leqq m\right\}=F(\alpha)
\end{aligned}
$$

where

$$
F(\alpha)= \begin{cases}0 & \text { if } \alpha<0, \\ \frac{12 \alpha}{\pi^{2}} & \text { if } 0<\alpha \leqq 1 / 2, \\ \frac{12 \alpha}{\pi^{2}}-\frac{12}{\pi^{2}} \int_{1 / 2}^{\alpha}\left(2-\frac{1}{y}-\frac{1-y}{y} \log \frac{y}{1-y}\right) d y \text { if } 1 / 2<\alpha \leqq 1, \\ 1 \quad \text { if } 1<\alpha .\end{cases}
$$

Proof. Firstly, 


$$
\lim _{k \rightarrow \infty} \frac{1}{k} \sum_{b=1}^{k} \frac{\Phi(b)}{b}=\frac{6}{\pi^{2}}
$$

Moreover, from (2.11) or (2.13) $\mu\left\{\left(S_{k}\right)\right\}=0$ for $k \leqq n / 2$ because $b_{1}<b_{2} \leqq k$ implies $b_{1}+b_{2}<n$. For $k>n / 2$ we have, following [3]( 4$)$,

$$
\begin{aligned}
\frac{2}{n} & \sum_{b_{1}=1}^{k} \sum_{\substack{b_{2}-\max \left(n-b_{1}, b_{1}+1\right) \\
\left(b_{1}, b_{2}\right)-1}}^{k} \frac{b_{1}+b_{2}-n}{b_{1} b_{2}} \\
& =\frac{2}{n}\left(\sum_{b_{1}=n-k}^{[(n-1) / 2]} \sum_{b_{2}=n-b_{1}}^{k}+\sum_{b_{1}=[(n+1) / 2]}^{k} \sum_{b_{2}-b_{1}+1}^{k}\right) \frac{b_{1}+b_{2}-n}{b_{1} b_{2}} \sum_{d \mid\left(b_{1}, b_{2}\right)} \mu(d) \\
& =T_{1}+T_{2} \text {, say, }
\end{aligned}
$$

where $\mu(\cdot)$ is the Möbius function [5, p. 234].

As an example we shall compute the asymptotic behaviour of $T_{1}$, the computation for $T_{2}$ being very similar. Changing the order of summation and putting $b_{2}=s d$ and

one has

$$
\{a\}=\text { smallest integer greater or equal to } a\left(^{(5)}\right.
$$

$$
T_{1}=\frac{2}{n} \sum_{b=n-k}^{[(n-1) / 2]} \frac{1}{b} \sum_{d \mid b} \mu(d) \sum_{s=\{(n-b) / d\}}^{[k / d]} \frac{b+s d-n}{s d} .
$$

However,

$$
\sum_{s=\mid(n-b) / d\}}^{[k / d]} \frac{b+s d-n}{s d}=\frac{b-n}{d} \log \frac{k}{n-b}+\frac{k-n+b}{d}+O(1) .
$$

Since [5, p. 235 and p. 260]

$$
\begin{gathered}
\sum_{d \mid b} \frac{\mu(d)}{d}=\frac{\Phi(b)}{b}, \quad \sum_{d \mid b} 1=O\left(b^{1 / 2}\right), \\
T_{1}=2 \sum_{b=n-k}^{[(n-1) / 2]} \frac{\Phi(b)}{b^{2}}\left(1-\frac{b}{n}\right) \log \left(1-\frac{b}{n}\right)+\frac{2 k}{n} \sum_{b=n-k}^{[(n-1) / 2]} \frac{\Phi(b)}{b^{2}} \\
+2\left(1+\log \frac{k}{n}\right) \frac{1}{n} \sum_{b=n-k}^{[(n-1) / 2]} \frac{\Phi(b)}{b}-2\left(1+\log \frac{k}{n}\right) \sum_{b=n-k}^{[(n-1) / 2]} \frac{\Phi(b)}{b^{2}} \\
+O\left(\frac{1}{n}+\frac{1}{k}\right) .
\end{gathered}
$$

Using the fact that $[5$, p. 268]

(5) This meaning of $\{a\}$ is used only in the next two formulae. 


$$
\sum_{b=1}^{n} \Phi(b)=\frac{3 n^{2}}{\pi^{2}}+O(n \log n)
$$

one obtains by partial summation as $n \rightarrow \infty, k \rightarrow \infty, k / n \rightarrow \alpha, 1 / 2 \leqq \alpha<1$,

$$
\begin{aligned}
\lim _{x / n \rightarrow \alpha} T_{1}=\frac{12}{\pi^{2}}\left[\int_{1-\alpha}^{1 / 2} \frac{1-y}{y}\right. & \log (1-y) d y-\alpha \log 2(1-\alpha) \\
& +(1+\log \alpha)(\alpha-1 / 2+\log 2(1-\alpha))] .
\end{aligned}
$$

In an entirely similar manner one obtains

$$
\begin{aligned}
\lim _{k / n \rightarrow \alpha} T_{2}=\frac{12}{\pi^{2}}\left[\alpha \log 2 \alpha-(\alpha-1 / 2)-\int_{1 / 2}^{\alpha} \frac{y-1}{y} \log y d y\right. \\
\left.+\log \alpha \int_{1 / 2}^{\alpha} \frac{y-1}{y} d y\right] .
\end{aligned}
$$

(2.17) and (2.18) imply

$$
\lim _{x / n \rightarrow \alpha}\left(T_{1}+T_{2}\right)=\frac{12}{\pi^{2}} \int_{1 / 2}^{\alpha}\left(2-\frac{1}{y}-\frac{1-y}{y} \log \frac{y}{1-y}\right) d y
$$

as one easily checks by comparing the derivatives of the right-hand sides of (2.17)-(2.19) as well as the values at $\alpha=1 / 2$.

From (2.9), (2.13), (2.15), (2.16), (2.19) one has

$$
\lim _{k / n \rightarrow \alpha} P\left\{t\left(x, \frac{1}{n}\right) \leqq k\right\}=F(\alpha),
$$

where $F(\alpha)$ is defined in (2.14). To prove the general relation

$$
\lim _{m \rightarrow \infty} P\left\{t\left(x, \frac{\alpha}{m}\right) \leqq m\right\}=F(\alpha)
$$

we notice, that if

$$
\frac{1}{n+1} \leqq \frac{\alpha}{m} \leqq \frac{1}{n}
$$

then for every $\xi$

$$
\iota\left(\xi, \frac{1}{n}\right) \leqq t\left(\xi, \frac{\alpha}{m}\right) \leqq t\left(\xi, \frac{1}{n+1}\right)
$$

Consequently also 


$$
P\left\{t\left(x, \frac{1}{n}\right) \leqq m\right\} \geqq P\left\{t\left(x, \frac{\alpha}{m}\right) \leqq m\right\} \geqq P\left\{t\left(x, \frac{1}{n+1}\right) \leqq m\right\}
$$

which, together with (2.20), (2.22) completes the proof of (2.21).

Till now we considered integers $k \leqq m$ for which $\langle k \xi\rangle \leqq \alpha / m$. In [2] one considered integers $k$ for which

$$
\langle k \xi\rangle \leqq \frac{\alpha}{k} .
$$

In particular, putting $(\alpha>0, c>1)$

$S(m, \alpha, c)=\{\xi: 0 \leqq \xi \leqq 1$, there exist integers $a, b$ for which

$$
m \leqq b \leqq m c,(a, b)=1,|b \xi-a| \leqq \alpha / b\} .
$$

Erdös, Szüsz and Turan [2] raised the question of finding

$$
\lim _{m \rightarrow \infty} \mu\{S(m, \alpha, c)\}
$$

if it exists at all. They found this limit for $\alpha \leqq c /\left(1+c^{2}\right)$ and gave bounds for $\mu\{S(m, \alpha, c)\}$ in several other cases. One has of course

$$
\left\{\xi: 0 \leqq \xi \leqq 1, m \leqq t\left(\xi, \frac{\alpha}{m c}\right) \leqq m c\right\} \subseteq S(m, \alpha, c)
$$

because

$$
\left|t\left(\xi, \frac{\alpha}{m c}\right) \xi-r\right| \leqq \frac{\alpha}{m c} \leqq \frac{\alpha}{t(\xi, \alpha / m c)}
$$

if $t(\xi, \alpha / m c) \leqq m c$, and at the same time (2.23) implies $(t(\xi, \alpha / m c), r)=1$ (cf. [3, p. 27]).

Consequently one has

$$
\liminf _{m \rightarrow \infty} \mu\{S(m, \alpha, c)\} \geqq F(\alpha)-F\left(\frac{\alpha}{c}\right) .
$$

Entirely obvious is the inclusion

$$
S(m, \alpha, c) \subseteq\left\{\xi: 0 \leqq \xi \leqq 1, t\left(\xi, \frac{\alpha}{m}\right) \leqq m c\right\},
$$

and therefore

$$
\limsup _{m \rightarrow \infty} \mu\{S(m, \alpha, c)\} \leqq F(\alpha c)
$$

(2.24) and (2.25) are improvements on the results of [2] for certain combinations of $\alpha$ and $c$. (2.25) however is only useful if $\alpha c<1$ (compare (2.14)). 
In this case, however, we can compute the limit of $\mu\{S(m, \alpha, c)\}$ exactly. The result is given by the next theorem.

TheOREM 2. If $\alpha \leqq c /\left(1+c^{2}\right)$, then

$$
\lim _{m \rightarrow \infty} \mu\{S(m, \alpha, c)\}=\frac{12 \alpha}{\pi^{2}} \log c
$$

If $c /\left(1+c^{2}\right) \leqq \alpha \leqq \min (1 / 2,1 / c)$, then

$$
\begin{aligned}
\lim _{m \rightarrow \infty} \mu\{S(m, \alpha, c)\}=\frac{12 \alpha}{\pi^{2}} \log c & -\frac{12}{\pi^{2}}\left(\alpha c+\frac{\alpha}{c}-\alpha \beta-\frac{\alpha}{\beta}\right. \\
& \left.+\alpha\left(\frac{1}{\beta}-\beta\right) \log \frac{c}{\beta}-\frac{1}{2}\left(\log \frac{c}{\beta}\right)^{2}\right),
\end{aligned}
$$

where

$$
\beta=\frac{1+\left(1-4 \alpha^{2}\right)^{1 / 2}}{2 \alpha}
$$

If $1 / 2 \leqq \alpha \leqq 1 / c$, then

$$
\lim _{m \rightarrow \infty} \mu\{S(m, \alpha, c)\}=\frac{12 \alpha}{\pi^{2}} \log c-\frac{12}{\pi^{2}}\left(\alpha c-2 \alpha+\frac{\alpha}{c}-\frac{1}{2}(\log c)^{2}\right) .
$$

Added in proof. Since this paper was written the following two references containing results related to this theorem have come to the author's attention: $\mathrm{P}$. Erdös, Some results on diophantine approximation, Acta Arithmetica 5 (1959), 359-369 and Richard P. Gosselin, On diophantine approximation and trigonometric polynomials, Pacific J. Math. 9 (1959), 1071-1081.

Proof. (2.26) is Theorem III of [2]. Instead of $I(a / b)$ we now define

$$
J\left(\frac{a}{b}\right)=\left[\frac{a}{b}-\frac{\alpha}{b^{2}}, \frac{a}{b}+\frac{\alpha}{b^{2}}\right] \quad \text { for } \frac{a}{b} \in F_{[m c]} .
$$

Then

$$
\begin{gathered}
S(m, \alpha, c)=\bigcup_{a / b \in F_{[m c ;} ; b \geq m} J\left(\frac{a}{b}\right) . \\
\sum_{a / b \in F_{[m c]} ; b \geq m} \mu\left\{J\left(\frac{a}{b}\right)\right\}=\sum_{m}^{[m c]} \frac{2 \alpha \Phi(b)}{b^{2}} \rightarrow \frac{12 \alpha}{\pi^{2}} \log c \quad(m \rightarrow \infty) .
\end{gathered}
$$

In addition, if $a_{1} / b_{1}$ and $a_{2} / b_{2}$ are two consecutive elements of $F_{\text {[mc] }}$ with $b_{1}, b_{2} \geqq m$, then 


$$
\left|\frac{a_{2}}{b_{2}}-\frac{a_{1}}{b_{1}}\right|=\frac{1}{b_{1} b_{2}} \geqq \max \left(\frac{\alpha}{b_{1}^{2}}, \frac{\alpha}{b_{2}^{2}}\right)
$$

by $\left[5\right.$, Theorem 28, p. 23] and the fact that $b_{1} / b_{2} \leqq c, b_{2} / b_{1} \leqq c, \alpha c \leqq 1$. Consequently $a_{1} / b_{1} \notin J\left(a_{2} / b_{2}\right), a_{2} / b_{2} \notin J\left(a_{1} / b_{1}\right)$ and therefore no $\xi$ can be in more than two intervals $J(a / b), b \geqq m, a / b \in F_{[m c]}$. If $\xi$ is in $J\left(a_{1} / b_{1}\right) \cap J\left(a_{2} / b_{2}\right)$ then $a_{1} / b_{1}$ and $a_{2} / b_{2}$ must be consecutive elements of $F_{[m c]}$. In this case

$$
\mu\left\{J\left(\frac{a_{1}}{b_{1}}\right) \cap J\left(\frac{a_{2}}{b_{2}}\right)\right\}=\max \left(0, \frac{\alpha}{b_{1}^{2}}+\frac{\alpha}{b_{2}^{2}}-\frac{1}{b_{1} b_{2}}\right) .
$$

Notice that for $\alpha \geqq 1 / 2$, always

$$
\frac{\alpha}{b_{1}^{2}}+\frac{\alpha}{b_{2}^{2}}-\frac{1}{b_{1} b_{2}} \geqq 0
$$

where as for $\alpha \leqq 1 / 2, b_{2}>b_{1}$

$$
\frac{\alpha}{b_{1}^{2}}+\frac{\alpha}{b_{2}^{2}}-\frac{1}{b_{1} b_{2}} \geqq 0
$$

only if

$$
\frac{b_{2}}{b_{1}} \geqq \beta=\frac{1+\left(1-4 \alpha^{2}\right)^{1 / 2}}{2 \alpha} .
$$

Thus, by (2.29) and (2.30)

$$
\mu\{S(m, \alpha, c)\}=\frac{12 \alpha}{\pi^{2}} \log c-\sum^{\prime \prime}\left(\frac{\alpha}{b_{1}^{2}}+\frac{\alpha}{b_{2}^{2}}-\frac{1}{b_{1} b_{2}}\right)+o(1)
$$

where $\sum^{\prime \prime}$ ranges over all pairs $a_{1} / b_{1}, a_{2} / b_{2}$ of consecutive elements of $F_{\text {[mc] }}$ with

$$
m \leqq b_{1}<b_{2} \leqq m c \quad \text { if } \alpha \geqq 1 / 2
$$

and with

$$
m \leqq b_{1}, \quad \beta b_{1} \leqq b_{2} \leqq m c \quad \text { if } \alpha \leqq 1 / 2 .
$$

It follows again from Lemma 1 in [3], that for given $b_{1}, b_{2}$ satisfying (2.31) if $\alpha \geqq 1 / 2$ or (2.32) if $\alpha \leqq 1 / 2$, there are exactly two pairs $\left(a_{1}, a_{2}\right)$ such that $a_{1} / b_{1}, a_{2} / b_{2}$ belong to $\sum^{\prime \prime}$ if $\left(b_{1}, b_{2}\right)=1$ and no such pairs if $\left(b_{1}, b_{2}\right)>1$. $\sum^{\prime \prime}$ can now be computed exactly as in Theorem 1 .

3. The distribution of $N(m, \gamma)$ in more dimensions. As we have seen in the last section, the study of the distribution of $\min _{1 \leq k \leq m}\langle k x\rangle$ was equivalent to finding $P\{N(m, \gamma)=0\}$ for appropriate $\gamma$. This raises the question of finding the complete distribution of $N(m, \gamma)$. Even though the methods of $\$ 2$ probably allow us to find the asymptotic distribution of $N(m, \alpha / m)$, it seems 
very hard to find the asymptotic distribution of $N(m, \gamma)-E N(m, \gamma)=N(m, \gamma)$ $-2 m \gamma$ after proper normalization, for fixed $\gamma$. The difficulty seems to be the "strong" dependence between the random variables $\langle k x\rangle, k=1,2, \cdots$. This shows a.o. in the fact that the distribution of $m^{-1 / 2}(N(m, \gamma)-2 m \gamma)$ does not approach a normal distribution, as it would if the random variables $\langle k x\rangle$ were strictly independent. In fact $m^{-1 / 2}$ is not at all the correct normalization factor [7]. It was suggested by $\mathrm{M}$. Kac in a discussion with the author that independence would approximately hold again for analogous random variables in high dimensions. This will be shown to be correct in a certain sense in the next theorem. The limiting distribution obtained in Theorem 3 is precisely the limiting distribution which would pertain if the $Y$ 's were strictly independent.

Let $x_{1}, x_{2}, \cdots$ be independent random variables, each with a uniform distribution on $[0,1]$. Define

$$
Y_{k}^{j}(\gamma)= \begin{cases}1 & \text { if }\left\langle k x_{1}\right\rangle \leqq \gamma,\left\langle k x_{2}\right\rangle \leqq \gamma, \cdots,\left\langle k x_{j}\right\rangle \leqq \gamma . \\ 0 & \text { otherwise }\end{cases}
$$

and

$$
N(m, \gamma, j)=\sum_{k=1}^{m} Y_{k}^{j}(\gamma) .
$$

$N(m, \gamma, j)$ is the number of indices $k \leqq m$ for which simultaneously $k x_{1}, \cdots$, $k x_{j}$ are closer than $\gamma$ to an integer.

TheOREm 3. If $0<\gamma<1 / 2$ is fixed and $p \rightarrow \infty, m \rightarrow \infty$ such that

$$
m(2 \gamma)^{p} \rightarrow \lambda>0
$$

then

$$
\lim P\{N(m, \gamma, p)=k\}=e^{-\lambda} \frac{\lambda^{k}}{k !}, \quad k=0,1, \cdots,
$$

that is, $N(m, \gamma, p)$ has asymptotically a Poisson distribution with mean $\lambda$.

Proof. Put for $k_{1}, \cdots, k_{r}$ pairwise different

$$
A_{\gamma}\left(k_{1}, \cdots, k_{r}\right)=P\left\{Y_{k_{1}}^{1}(\gamma)=\cdots=Y_{k_{r}}^{1}(\gamma)=1\right\} .
$$

$\gamma$ will be fixed throughout $(0<\gamma<1 / 2)$ and the index or subscript $\gamma$ will only be written explicitly when necessary to avoid confusion. Since the $x_{1}, x_{2}, \ldots$ are independent one has clearly

$$
\begin{aligned}
P\left\{Y_{k_{1}}^{j}=\cdots=Y_{k_{r}}^{j}=1\right\} & =\left(P\left\{Y_{k_{1}}^{1}=\cdots=Y_{k_{r}}^{1}=1\right\}\right)^{j} \\
& =A^{j}\left(k_{1}, \cdots, k_{r}\right) .
\end{aligned}
$$


Moreover,

$$
\begin{array}{r}
E(N(m, \gamma, j)(N(m, \gamma, j)-1)(N(m, \gamma, j)-2) \cdots(N(m, \gamma, j)-r+1)) \\
=E \sum_{k(r)} Y_{k_{1}}^{j} \cdots Y_{k_{r}}^{j}=\sum_{k(r)} A^{j}(k(r)) .
\end{array}
$$

We adopted here the convention to write $k(r)$ for a generic $r$-tuple $\left(k_{1}, \cdots, k_{r}\right)$ of different integers $k_{i}, 1 \leqq k_{i} \leqq m$, and to include in $\sum_{k(r)}$ all such $r$-tuples in the $r$ ! orders in which they can appear. This convention will be used through the remainder of this section.

One easily checks that if $N$ has a Poisson distribution with mean $\lambda$, then its $r$ th factorial moment,

$$
E(N(N-1)(N-1) \cdots(N-r+1))=\lambda^{r}, \quad r=1,2, \cdots .
$$

Conversely, if $\{N(p)\}$ is a sequence of random variables for which

$\lim _{p \rightarrow \infty} E(N(p)(N(p)-1)(N(p)-2) \cdots(N(p)-r+1))=\lambda^{r}, \quad r=1,2, \cdots$ then the limiting distribution $(p \rightarrow \infty)$ of $N(p)$ is a Poisson distribution with mean $\lambda$ (by [9, p. 185 C; 6, p. 1154.30, p. 1094.21$]$ ).

Theorem 3 will therefore be proved, if we can show

$$
\lim \sum_{k(r)} A^{p}(k(r))=\lambda^{r}, \quad r=1,2, \cdots,
$$

or equivalently, by (3.1)

$$
\lim \frac{\sum_{k(r)}\left(\frac{A_{\gamma}(k(r))}{(2 \gamma)^{r}}\right)^{p}}{m(m-1) \cdots(m-r+1)}=1, \quad r=1,2, \cdots .
$$

We shall prove (3.5) by induction on $r$.

Let us put

$$
\nu_{r}^{(j)}(\gamma)=\frac{\sum_{k(r)}\left(\frac{A_{\gamma}(k(r))}{(2 \gamma)^{r}}\right)^{j}}{m(m-1) \cdots(m-r+1)} .
$$

Then

$$
\nu_{1}^{(p)}(\gamma)=\frac{\sum_{k=1}^{m} A_{\gamma}^{p}(k)}{m(2 \gamma)^{p}}=\frac{\sum_{k=1}^{m}\left(P\left\{Y_{k}^{1}(\gamma)=1\right\}\right)^{p}}{m \cdot(2 \gamma)^{p}}=1
$$

since

$$
P\left\{Y_{k}^{1}(\gamma)=1\right\}=P\left\{\left\langle k x_{1}\right\rangle \leqq \gamma\right\}=2 \gamma .
$$


Thus (3.5) holds for $r=1$. The remaining details will be split up in a number of lemmas. $C_{i}, i=1,2, \cdots$, will always stand for some finite, positive constant which depends on $\gamma, r, \lambda$ only.

LEMMA 1. There exists a $C_{1}$ such that for any sequence of $s$ positive integers $k_{1}<k_{2}<\cdots<k_{\text {s }}$

$$
E\left(\sum_{j=1}^{\infty} Y_{k_{j}}^{1}(\gamma)-2 s \gamma\right)^{2} \leqq \frac{s}{2}\left(C_{1}+(\log s)^{2}\right) .
$$

$C_{1}$ does not depend on $s$.

Proof $\left({ }^{3}\right)$.

$$
\begin{aligned}
\left.E\left(\sum_{j=1}^{\dot{1}} Y_{k_{j}}^{1}-2 s \gamma\right)\right)^{2} & =\operatorname{var}\left(\sum_{j=1}^{s} Y_{k_{j}}^{1}\right) \\
& =s 2 \gamma(1-2 \gamma)+2 \sum_{1 \leq i<j \leq s}\left(A\left(k_{i}, k_{j}\right)-4 \gamma^{2}\right) .
\end{aligned}
$$

It was proved in $[8$, p. 217 line 4$]$ that

$$
\left|A_{\gamma}\left(k_{i}, k_{j}\right)-4 \gamma^{2}\right| \leqq \frac{\left(k_{i}, k_{j}\right)^{2}}{k_{i} \cdot k_{j}}=\frac{1}{k_{i}^{\prime} \cdot k_{j}^{\prime}}
$$

where $\left(k_{i}, k_{j}\right)=$ greatest common divisor of $k_{i}$ and $k_{j}$ and

$$
k_{i}^{\prime}=\frac{k_{i}}{\left(k_{i}, k_{j}\right)}, \quad k_{j}^{\prime}=\frac{k_{j}}{\left(k_{i}, k_{j}\right)} .
$$

Notice that for any pair of integers $a<b$ there are at most $s$ possible pairs $k_{i}<k_{j}$ such that $k_{i}^{\prime}=a, k_{j}^{\prime}=b$ and hence, for any positive integer $c$ there are at most $s d(c) / 2$ pairs $k_{i}<k_{j}$ with $k_{i}^{\prime} \cdot k_{j}^{\prime}=c(d(c)=$ number of divisors of $c)$. Therefore,

$$
\sum_{1 \leq i<j \leq s}\left|A_{j}\left(k_{i}, k_{j}\right)-4 \gamma^{2}\right| \leqq \frac{s}{2} \sum_{c=1}^{c_{0}} \frac{d(c)}{c}
$$

where $c_{0}$ is the smallest integer for which

$$
\frac{s}{2} \sum_{c=1}^{c_{0}} d(c) \geqq \frac{s(s-1)}{2} .
$$

In fact there are $s(s-1) / 2$ pairs $k_{i}<k_{j}$ and we have replaced each $1 / k_{i}^{\prime} k_{j}^{\prime}$ by some $1 / c$ with $1 / k_{i}^{\prime} k_{j}^{\prime} \leqq 1 / c$. Since

$$
\sum_{c=1}^{n} d(c)=n \log n+O(n)
$$

[5, Theorem 320 , p. 264], 


$$
\frac{s}{2} \sum_{c=1}^{c_{0}} \frac{d(c)}{c} \leqq \frac{s}{2} \sum_{c=1}^{28 / \log s} \frac{d(c)}{c} \leqq \frac{s}{4}(\log s)^{2}
$$

for sufficiently large $s$. This together with (3.7) and (3.8) implies the lemma.

The estimate of [8] for $\left|A\left(k_{i}, k_{j}\right)-4 \gamma^{2}\right|$ would suffice to prove directly $\nu_{2}^{(p)}(\gamma) \rightarrow 1$, and one might want to follow LeVeque's method [8] of estimating $A_{\gamma}\left(k_{1}, k_{j}\right)$ also for $A_{\gamma}\left(k_{1}, \cdots, k_{r}\right)$ in order to prove (3.5) directly for all $r$. This would require an estimate of

$$
A \gamma\left(k_{1}, \cdots, k_{r}\right)=\int_{0}^{1} d \xi \prod_{t=1}^{r}\left(2 \gamma+\frac{2}{\pi} \sum_{n_{t}=1}^{\infty} \frac{\sin 2 \pi n_{t} \gamma \cos 2 \pi n_{t} k_{t} \xi}{n_{t}}\right) .
$$

We have been unable to follow this direct procedure and are forced to prove (3.5) by a detour.

Lемма 2. If $X$ is any random variable satisfying

$$
E(X-a)^{2} \leqq c^{2}
$$

and

$$
0 \leqq X \leqq b \quad \text { with probability one, }
$$

then, there exists constants $C_{2}$, independent of the distribution of $X$ such that

$$
\begin{aligned}
|E X-a| & \leqq c, \\
\int_{2 a}^{\infty} y^{r} d P\{X \leqq y\} & \leqq C_{2}(r) b^{r-2} c^{2}, \quad r=3,4, \cdots,
\end{aligned}
$$

and

$$
\left|E X^{r}-(E X)^{r}\right| \leqq C_{2}(r) b^{r-2} c^{2}, \quad r=2,3,4, \cdots .
$$

Proof. Put $E X=\mu$. Then

$$
\begin{aligned}
c^{2} & \geqq E(X-a)^{2}=E(X-\mu+\mu-a)^{2}=E(X-\mu)^{2}+(\mu-a)^{2} \\
& \geqq(E X-a)^{2} .
\end{aligned}
$$

This proves (3.12). As for (3.13) and (3.14) we use the one sided analogue of Tchebyshev's inequality. For $\lambda \geqq 0$,

$$
\begin{aligned}
P\{X-a \geqq \lambda\} & \leqq \frac{1}{\left(\lambda^{2}+c^{2}\right)^{2}} \int_{a}^{\infty}\left(\lambda(y-a)+c^{2}\right)^{2} d P\{X \leqq y\} \\
& \leqq \frac{2}{\left(\lambda^{2}+c^{2}\right)^{2}} \int_{a}^{\infty}\left(\lambda^{2}(y-a)^{2}+c^{4}\right) d P\{X \leqq y\} \\
& \leqq \frac{2}{\left(\lambda^{2}+c^{2}\right)^{2}}\left(\lambda^{2} c^{2}+c^{4}\right)=\frac{2 c^{2}}{\lambda^{2}+c^{2}}
\end{aligned}
$$


Putting

$$
G(\lambda)=\left\{\begin{array}{cl}
0 & \text { if } \lambda \leqq c, \\
1-\frac{2 c^{2}}{\lambda^{2}+c^{2}} & \text { if } c \leqq \lambda<b-a, \\
1 & \text { if } b-a \leqq \lambda,
\end{array}\right.
$$

one has by (3.11) and (3.16)

$$
1-P\{X \leqq a+\lambda\} \leqq 1-G(\lambda) .
$$

Consequently, for $r \geqq 3$

$$
\begin{aligned}
\int_{2 a}^{\infty} y^{r} d P\{X \leqq y\} & =\int_{2 a}^{b+} y^{r} d P\{X \leqq y\} \\
& =-\left.(1-P\{X \leqq y\}) y^{r}\right|_{2 a} ^{b}+r \int_{2 a}^{b} y^{r-1}(1-P\{X \leqq y\}) d y \\
& =(1-P\{X \leqq 2 a\})(2 a)^{r}+r \int_{2 a}^{b} y^{r-1}(1-P\{X \leqq y\}) d y \\
& \leqq(2 a)^{r}(1-G(a))+r \int_{a}^{b-a}(z+a)^{r-1}(1-G(z)) d z \\
& \leqq(2 a)^{r} \frac{2 c^{2}}{a^{2}}+2^{r-1} r \int_{a}^{b-a} z^{r-1} \cdot \frac{2 c^{2}}{z^{2}+c^{2}} d z .
\end{aligned}
$$

This implies (3.13) when $2 a \leqq b$. When $2 a>b$ (3.13) becomes trivial by (3.11).

(3.14) is proved similar to (3.13). By (3.11), $0 \leqq \mu \leqq b$ and by (3.15) $E(X-\mu)^{2} \leqq c^{2}$. Thus, with $a$ replaced by $\mu$ one has from (3.16)

$$
1-P\{X \leqq \mu+\lambda\} \leqq 1-G(\lambda)
$$

and similarly with $a$ replaced by $b-\mu$

$$
P\{X \leqq \mu-\lambda\} \leqq 1-G(\lambda) .
$$

Now, since $E(X-\mu)$ equals zero,

$$
\begin{aligned}
E X^{r}=E(\mu+X-\mu)^{r}=\mu^{r} & +\left(\begin{array}{l}
r \\
2
\end{array}\right) \mu^{r-2} E(X-\mu)^{2} \\
& +\sum_{j=3}^{r}\left(\begin{array}{l}
r \\
j
\end{array}\right) \mu^{r-j} E(X-\mu)^{i} .
\end{aligned}
$$

By (3.17), for $j \geqq 3$

$$
\int_{y \geq \mu}(y-\mu)^{j} d P\{X \leqq y\} \leqq \int_{0}^{b-\mu} z^{j} d G(z) \leqq C_{3}(j) b^{j-2} c^{2}
$$


and by (3.18)

$$
\int_{y \leqq \mu}|y-\mu|^{j} d P\{X \leqq y\} \leqq C_{3}(j) b^{j-2} c^{2} .
$$

(3.14) follows from (3.19)-(3.21).

An important consequence of Lemma 1 and Lemma 2 is the following:

LEMмa 3. If $p \rightarrow \infty, m \rightarrow \infty$ such that $m(2 \gamma)^{p} \rightarrow \lambda$, then for some $C_{4}$, depending on $\gamma, r, \lambda$ only, and $j \leqq p$

$$
\exp \left(-C_{4}(2 \gamma)^{p-j}\left(1+(p-j)^{2}\right) \leqq \nu_{r}^{(j)}(\gamma) \leqq \exp \left(C_{4}(2 \gamma)^{p-j}\left(1+(p-j)^{2}\right)\right.\right.
$$

Proof. As we remarked already, Lemma 3 is obvious for $r=1$, and we may assume $r \geqq 2$.

$$
\begin{aligned}
\nu_{r}^{(j)}(\gamma) & =\frac{E(N(m, \gamma, j) \cdots(N(m, \gamma, j)-r+1))}{m(m-1) \cdots(m-r+1)(2 \gamma)^{r j}} \\
& \leqq \frac{E N^{r}(m, \gamma, j)}{m(m-1) \cdots(m-r+1)(2 \gamma)^{r j}}
\end{aligned}
$$

Denote by $F\left(k_{1}, \cdots, k_{s} ; u\right)$ the event

$Y_{k_{1}}^{u}=Y_{k_{2}}^{u}=\cdots=Y_{k_{s}}^{u}=1$ whereas $Y_{k}^{u}=0$ if $k \neq k_{i}, \quad i=1,2, \cdots, s$.

Then, dropping the indices $m$ and $\gamma$ for this proof,

$E N^{r}(j)=\sum_{1 \leq k_{1}<k_{2} \cdots<k_{s} \leq m} P\left\{F\left(k_{1}, \cdots, k_{s} ; j-1\right)\right\} E\left(N^{r}(j) \mid F\left(k_{1}, \cdots, k_{s} ; j-1\right)\right)$.

However,

$$
E\left(N^{r}(j) \mid F\left(k_{1}, \cdots, k_{s} ; j-1\right)\right)=E\left(\sum_{t=1}^{s} Y_{k_{t}}^{1}\right)^{r}
$$

because if $Y_{k_{1}}^{j-1}=1$, then $Y_{k_{1}}^{f}=1$ if and only if $\left\langle k_{1} x_{j}\right\rangle \leqq \gamma$ and, if $Y_{k_{1}}^{j-1}=0$ then $Y_{k_{1}}^{f}$ always equals zero. Hence,

$$
\begin{aligned}
E\left(N(j) \mid F\left(k_{1}, \cdots, k_{s} ; j-1\right)\right) & \leqq 2 \gamma s \\
\operatorname{var}\left(N(j) \mid F\left(k_{1}, \cdots, k_{s} ; j-1\right)\right) & \leqq \frac{s}{2}\left(C_{1}+(\log s)^{2}\right) \quad(\text { Lemma 1) }
\end{aligned}
$$

and since $0 \leqq N(j) \leqq s$ if $F\left(k_{1}, \cdots, k_{s} ; j-1\right)$ occurs, $\left.E\left(N^{r}(j) \mid F\left(k_{1}, \cdots, k_{s}\right) ; j-1\right)\right) \leqq(2 \gamma)^{r} s^{r}+C_{2}(r) \frac{s^{r-1}}{2}\left(C_{1}+(\log s)^{2}\right)(\operatorname{by}(3.14))$.

If we take into account that 


$$
N(j-1)=s \quad \text { if } F\left(k_{1}, \cdots, k_{s} ; j-1\right)
$$

occurs one has

$$
\begin{aligned}
E N^{r}(j) \leqq & \sum_{1 \leq k_{1}<\cdots<k_{s} \leq m} P\left\{F\left(k_{1}, \cdots, k_{s} ; j-1\right\}\right. \\
& \cdot\left[(2 \gamma)^{r} N^{r}(j-1)+\frac{C_{2}}{2} N^{r-1}(j-1)\left(C_{1}+(\log N(j-1))^{2}\right)\right] \\
= & (2 \gamma)^{r} E N^{r}(j-1)+\frac{C_{2} C_{1}}{2} E N^{r-1}(j-1) \\
+ & \frac{C_{2}}{2} E N^{r-1}(j-1)(\log N(j-1))^{2} .
\end{aligned}
$$

Applying Jensen's inequality $[9$, p. 156, c, $]$ twice gives

(3.26) $E N^{r-1}(j-1) \leqq\left(E N^{r}(j-1)\right)^{(r-1) / r} \leqq \frac{E N^{r}(j-1)}{E N(j-1)}=\frac{E N^{r}(j-1)}{m \cdot(2 \gamma)^{j-1}}$.

The last term of (3.25) is estimated first by using Hölder's inequality [9, p. 156]

$$
\begin{aligned}
E N^{r-1}(j-1)(\log N(j-1))^{2} & \\
\leqq(2 r-1)^{2} E N^{r-1}(j-1)+ & \int_{\exp (2 r-1)}^{\infty} y^{r-1}(\log y)^{2} d P\{N(j-1) \leqq y\} \\
\leqq(2 r-1)^{2} E N^{r-1}(j-1)+ & \left(\int_{\exp (2 r-1)}^{\infty} y^{r} d P\{N(j-1) \leqq y\}\right)^{(r-1) / r} \\
& .\left(\int_{\exp (2 r-1)}^{\infty}(\log y)^{2 r} d P\{N(j-1) \leqq y\}\right)^{1 / r} .
\end{aligned}
$$

Since $d^{2}(\log y)^{2 r} / d y^{2} \leqq 0$ for $y \geqq \exp (2 r-1)$, Jensen's inequality [4, Theorem 95 , p. $77 ; 9$, p. 159 , e] implies

$$
\begin{aligned}
& \int_{\exp (2 r-1)}^{\infty}(\log y)^{2 r} d P\{N(j-1) \leqq y\} \\
& (3.27) \leqq P\{N(j-1) \geqq \exp (2 r-1)\}\left(\log \frac{\int_{\exp (2 r-1)}^{\infty} y d P\{N(j-1) \leqq y\}}{\int_{\exp (2 r-1)}^{\infty} d P\{N(j-1) \leqq y\}}\right)^{2 r} \\
& \quad=O(\log E N(j-1))^{2 r} \\
& \quad=O\left(\log m(2 \gamma)^{j}\right)^{2 r} .
\end{aligned}
$$


Applying again (3.26) to $E N^{r-1}(j-1)$ and combining (3.25)-(3.27) one obtains for some $C_{5}(\gamma, r)$

$$
\begin{aligned}
E N^{r}(j) & \leqq(2 \gamma)^{r} E N^{r}(j-1)\left(1+C_{5} \frac{1+\left(\log m(2 \gamma)^{j}\right)^{2}}{m \cdot(2 \gamma)^{j}}\right) \\
& \leqq(2 \gamma)^{r} E N^{r}(j-1) \exp \left(C_{5} \frac{1+\left(\log m(2 \gamma)^{j}\right)^{2}}{m(2 \gamma)^{j}}\right)
\end{aligned}
$$

This also holds for $j=1$ if we put $N(0)=m$ with probability one. Thus by induction on $j$

$$
E N^{r}(j) \leqq(2 \gamma)^{r i} m^{r} \exp \left(C_{5} \sum_{n=1}^{j} \frac{1+\left(\log m(2 \gamma)^{n}\right)^{2}}{m(2 \gamma)^{n}}\right),
$$

which immediately gives the right-hand inequality of (3.22) if we take into account $m(2 \gamma)^{p} \rightarrow \lambda$.

In the same way one shows $\left({ }^{6}\right)$

$$
E N^{r}(j) \geqq(2 \gamma)^{r i} m^{r} \exp \left(-C_{5} \sum_{n=1}^{j} \frac{1+\left(\log m(2 \gamma)^{n}\right)^{2}}{m(2 \gamma)^{n}}\right)
$$

which implies the left-hand inequality of (3.22) because

$$
E N(N-1) \cdots(N-r+1)=E N^{r}+O\left(E \sum_{k=0}^{r-1} N^{k}\right) .
$$

Lemma 4. There exists a constant $C_{6}(r, \gamma)<\infty$ such that

$$
A_{\gamma}^{-1}(k(r)) \leqq C_{6}(r, \gamma)
$$

Proof.

$$
2 \gamma+\frac{2}{\pi} \sum_{n=1}^{\infty} \frac{\sin 2 \pi n \gamma \cos 2 \pi n \xi}{n}= \begin{cases}1 & \text { if }\langle\xi\rangle<\gamma \\ 0 & \text { if }\langle\xi\rangle>\gamma\end{cases}
$$

(cf. [8]). Hence

$$
A_{\gamma}\left(k_{1}, \cdots, k_{r}\right)=\int_{0}^{1} d \xi \prod_{t=1}^{r}\left(2 \gamma+\frac{2}{\pi} \sum_{n_{t}=1}^{\infty} \frac{\sin 2 \pi n_{t} \gamma \cos 2 \pi n_{t} k_{t} \xi}{n_{t}}\right) .
$$

Since

(8) We shall use the left-hand inequality of (3.22) only for $j \leqq(2 p+6) / 3$. For this range the argument in the next few lines suffices but not for all $j \leqq p$. However, the left-hand inequality of (3.22) is true for all $j \leqq p$. The same remark applies to the left-hand inequality of (3.45). 


$$
\sum_{n=M+1}^{\infty} \frac{\sin 2 \pi n \gamma \cos 2 \pi n k \xi}{n}
$$

is bounded uniformly in $M, k, \xi$ (being the tail of the Fourier series of a function of bounded variation $[10$, p. 408]) and since

$$
\int_{0}^{1}\left|\sum_{n=M+1}^{\infty} \frac{\sin 2 \pi n \gamma \cos 2 \pi n k \xi}{n}\right| d \xi=\int_{0}^{1}\left|\sum_{n=M+1}^{\infty} \frac{\sin 2 \pi n \gamma \cos 2 \pi n \xi}{n}\right| d \xi
$$

tends to zero as $M \rightarrow \infty$ (uniformly in $k$ ), it is possible to choose $M=M(\gamma, t)$ such that

$$
\begin{aligned}
A\left(k_{1}, \cdots, k_{r}\right) & \\
& \geqq \int_{0}^{1} d \xi \prod_{t=1}^{r}\left(2 \gamma+\frac{2}{\pi} \sum_{n_{t}=1}^{M} \frac{\sin 2 \pi n_{t} \gamma \cos 2 \pi n_{t} k_{t} \xi}{n_{t}}\right)-\frac{(2 \gamma)^{r}}{2} \\
& =\frac{(2 \gamma)^{r}}{2}+\sum_{j=1}^{r}(2 \gamma)^{r-i}\left(\frac{2}{\pi}\right)^{j} \sum_{1 \leq t_{i} \leq r} \int_{0}^{1} d \xi \prod_{i=1}^{j} \frac{\sin 2 \pi n_{t_{i}} \gamma \cos 2 \pi n_{t_{i}} k_{t_{i} \xi}}{n_{t_{i}}} .
\end{aligned}
$$

The last integrals can only be different from zero if some relation

$$
\sum_{t=1}^{r} \epsilon_{t} n_{t} k_{t}=0, \quad \epsilon_{t}=0,+1,-1
$$

holds, with at least two $\epsilon$ 's different from zero. We can now prove the lemma by induction on $r . A_{\gamma}\left(k_{1}\right)=2 \gamma$ i.e. $C_{6}(1, \gamma)=(2 \gamma)^{-1}$ satisfies (3.28) for $r=1$. Let (3.28) be proved already for $r-1$ and assume there exists a sequence $\left(k_{1}^{(n)}, \cdots, k_{r}^{(n)}\right) n=1,2, \cdots$ of $r$-tuples $k^{(n)}(r)$ such that

$$
\lim _{n \rightarrow \infty} A\left(k^{(n)}(r)\right)=0 \text {. }
$$

By virtue of (3.29) and (3.30) we may assume, if necessary by selecting a subsequence and rearranging the indices, that for some fixed $\epsilon_{1}, \cdots, \epsilon_{r}$ and $n_{1}, \cdots, n_{r} \leqq M(\gamma, r)$ and all $n$

$$
\sum_{t=1}^{r} \epsilon_{t} n_{t} k_{t}^{(n)}=0, \quad \epsilon_{r-1} \epsilon_{r} \neq 0 .
$$

In this case, however, one has for any $\xi$ satisfying

$$
\left\langle k_{j}^{(n)} \xi\right\rangle \leqq \frac{\gamma}{r M}, \quad j=1, \cdots, r-1,
$$

also

$$
\left\langle n_{r} k_{j}^{(n)} \xi\right\rangle \leqq \frac{\gamma}{r} \leqq \gamma, \quad j=1, \cdots, r-1
$$


and

$$
\left\langle n_{r} k_{r}^{(n)} \xi\right\rangle=\left\langle\sum_{t=1}^{r-1} \epsilon_{t} n_{t} k_{t}^{(n)} \xi\right\rangle \leqq \frac{(r-1) M}{r M} \gamma \leqq \gamma .
$$

Consequently,

$$
\begin{aligned}
A_{\gamma}\left(n_{r} k_{1}^{(n)}, n_{r} k_{2}^{(n)}, \cdots, n_{r} k_{r}^{(n)}\right) & \geqq A_{\gamma / r M}\left(k_{1}^{(n)}, \cdots, k_{r-1}^{(n)}\right) \\
& \geqq C_{6}\left(r-1, \frac{\gamma}{r M}\right)^{-1} .
\end{aligned}
$$

But $A\left(k_{1}^{(n)}, \cdots, k_{r}^{(n)}\right)=A\left(n_{r} k_{1}^{(n)}, \cdots, n_{r} k_{r}^{(n)}\right)$ because $n_{r} x$ has a uniform distribution modulo one if $x$ has. (3.31) contradicts (3.30) which proves the lemma.

LEMMA 5. There exists a positive constant $C_{7}(r, \gamma)$ for every $r \geqq 2$, such that for every $k(r)=\left(k_{1}, \cdots, k_{r}\right)$

$$
\frac{A_{\gamma}(k(r))}{A_{\gamma}\left(k_{1}, \cdots, k_{j-1}, k_{j+1}, \cdots, k_{r}\right)} \leqq 1-C_{7}(r, \gamma)<1
$$

for some $1 \leqq j \leqq r$.

Proof. For shortness put $k(r, j)=\left(k_{1}, \cdots, k_{j-1}, k_{j+1}, \cdots, k_{r}\right)$. By the definition of $A$

$$
\begin{aligned}
\frac{A(k(r))}{A(k(r, j))} & =P\left\{Y_{k_{j}}^{1}=1 \mid Y_{k_{i}}^{1}=1,1 \leqq i \leqq r, i \neq j\right\} \\
& =1-A^{-1}(k(r, j)) P\left\{Y_{k_{j}}^{1}=0, Y_{k_{i}}^{1}=1,1 \leqq i \leqq r, i \neq j\right\}
\end{aligned}
$$

It therefore suffices to prove

$$
\begin{array}{rlr}
B(k(r, j)) & =P\left\{Y_{k_{j}}^{1}=0, Y_{k_{i}}^{1}=1,1 \leqq i \leqq r, i \neq j\right\} \\
& \geqq C_{8}(r, j)>0 \quad \text { for some } 1 \leqq j \leqq r
\end{array}
$$

where $C_{8}(r, j)$ does not depend on $k(r)$.

Assume (3.33) does not hold and that $k^{(n)}(r), n=1,2, \cdots$, is a sequence of $r$-tuples for which

$$
\lim _{n \rightarrow \infty} B\left(k^{(n)}(r, j)\right)=0, \quad j=1, \cdots, r .
$$

Without loss of generality we may rearrange the indices and select a subsequence such that

$$
k_{1}^{(n)}<k_{2}^{(n)}<\cdots<k_{r}^{(n)}
$$
for all $n$

and 


$$
\lim _{n \rightarrow \infty} \frac{k_{r}^{(n)}}{k_{i}^{(n)}} \geqq 1
$$

exists for $i=1, \cdots, r$

(infinity is allowed as a limit in (3.36)). Let

$$
\lim _{n \rightarrow \infty} \frac{k_{r}^{(n)}}{k_{i}^{(n)}}=1 \quad \text { for } i=s+1, \cdots, r
$$

while

$$
\lim _{n \rightarrow \infty} \frac{k_{r}^{(n)}}{k_{i}^{(n)}}>1
$$

for $i=1, \cdots, s$.

By deleting some $n$ 's we can assume

$$
\frac{k_{j}^{(n)}}{k_{i}^{(n)}} \geqq 1+\delta,
$$

$i=1, \cdots, s, \quad j=s+1, \cdots, r$ for all $n$ and some $0<\delta \leqq 1$.

Let $g(\xi)$ be the fractional part of $\xi$ minus the integer closest to $\xi$. More precisely

$$
\begin{aligned}
g(\xi) & =\left\{\begin{array}{cl}
\xi & \text { if } 0 \leqq \xi \leqq 1 / 2, \\
\xi-1 & \text { if } 1 / 2<\xi \leqq 1,
\end{array}\right. \\
g(\xi+1) & =g(\xi) .
\end{aligned}
$$

One has

$$
P\left\{\left|g\left(k_{i} x\right)-g\left(k_{j} x\right)\right| \leqq \eta\right\} \leqq P\left\{\left|g\left(\left(k_{i}-k_{j}\right) x\right)\right| \leqq \eta\right\} \leqq 2 \eta
$$

so that for

$$
\eta=\min \left[\left(4\left(\begin{array}{l}
r \\
2
\end{array}\right) C_{6}\left(r, \frac{\gamma \delta}{4(1+\delta)}\right)\right)^{-1}, \frac{1}{\gamma}-2, \frac{\delta}{(2+\delta)}\right]
$$

$$
\begin{aligned}
P\left\{\left|g\left(k_{i} x\right)\right|\right. & \left.\leqq \frac{\gamma \delta}{4(1+\delta)},\left|g\left(k_{i} x\right)-g\left(k_{j} x\right)\right| \geqq \eta, 1 \leqq i, j \leqq r, i \neq j\right\} \\
& \geqq A_{\gamma \delta(4+4 \delta)^{-1}(k(r))-\sum_{1 \leqq i<j \leqq r} P\left\{\left|g\left(k_{i} x\right)-g\left(k_{j} x\right)\right| \leqq \eta\right\}} \\
& \geqq C_{6}^{-1}\left(r, \frac{\gamma \delta}{4(1+\delta)}\right)-\left(\begin{array}{c}
r \\
2
\end{array}\right) \cdot 2 \eta \\
& \geqq \frac{1}{2} C_{6}^{-1}\left(r, \frac{\gamma \delta}{4(1+\delta)}\right)>0 .
\end{aligned}
$$


There exists therefore a $\xi$, depending on $n$ and satisfying

$$
\left|g\left(k_{i}^{(n)} \xi\right)\right| \leqq \frac{\gamma \delta}{4(1+\delta)},\left|g\left(k_{i}^{(n)} \xi\right)-g\left(k_{j}^{(n)} \xi\right)\right| \geqq \eta,
$$

$1 \leqq i, j \leqq r, i \neq j$. Let $j_{1}$ be determined by

$$
\left.g\left(k_{j_{1}}^{(n)} \xi\right)=\max _{8+1 \leqq j \leqq r} g\left(k_{j}^{(n)} \xi\right) \quad \text { (thus } j_{1} \geqq s+1\right)
$$

and put

$$
\bar{\xi}=\frac{\gamma(1+\eta / 2)-g\left(k_{j_{1}}^{(n)} \xi\right)}{k_{j_{1}}^{(n)}} \leqq \frac{\gamma(1+\eta / 2+\delta / 4(1+\delta))}{k_{j_{1}}^{(n)}}
$$

Then

$$
g\left(k_{j_{1}}^{(n)} \xi\right)+k_{j_{1}}^{(n)} \bar{\xi}=\gamma\left(1+\frac{\eta}{2}\right) \leqq \frac{1}{2}
$$

On the other hand, by (3.38), (3.41) and (3.42), for any $i \leqq s$

$$
g\left(k_{i}^{(n)} \xi\right)+k_{i}^{(n)} \bar{\xi} \leqq \frac{\gamma \delta}{4(1+\delta)}+\frac{\gamma(1+\eta / 2+\delta / 4(1+\delta))}{1+\delta} \leqq \gamma\left(1-\frac{\eta}{2}\right),
$$

and for any $i \geqq s+1, i \neq j_{1}$

$$
\begin{aligned}
g\left(k_{i}^{(n)} \xi\right) & +k_{i}^{(n)} \bar{\xi} \leqq g\left(k_{j_{1}}^{(n)} \xi\right)-\eta+k_{j_{1}}^{(n)} \bar{\xi}+\left(k_{i}^{(n)}-k_{j_{1}}^{(n)}\right) \bar{\xi} \\
& \leqq \gamma\left(1+\frac{\eta}{2}\right)-\eta+\frac{\left|k_{i}^{(n)}-k_{j_{1}}^{(n)}\right|}{k_{j_{1}}^{(n)}} \gamma\left(1+\frac{\eta}{2}+\frac{\delta}{4(1+\delta)}\right) .
\end{aligned}
$$

Since, for $i, j_{1} \geqq s+1$

$$
\frac{k_{i}^{(n)}}{k_{j_{1}}^{(n)}} \rightarrow 1
$$

the last member of (3.44) will eventually also be less than $\gamma(1-\eta / 2)$. Thus for sufficiently large $n$

$$
g\left(k_{j_{1}}^{(n)} \xi\right)+k_{j_{1}}^{(n)} \xi=g\left(k_{j_{1}}^{(n)}(\xi+\bar{\xi})\right)=\gamma\left(1+\frac{\eta}{2}\right)
$$

and by (3.41)-(3.44)

$$
-\frac{\gamma \delta}{4(1+\delta)} \leqq g\left(k_{i}^{(n)}(\xi+\bar{\xi})\right) \leqq \gamma\left(1-\frac{\eta}{2}\right) \quad \text { if } i \neq j_{1} .
$$

If now 


$$
\left\langle k_{i}^{(n)} t\right\rangle<\gamma \frac{\eta}{2}, \quad i=1, \cdots, r,
$$

then

$$
\left\langle k_{i}^{(n)}(\xi+\bar{\xi}+t)\right\rangle \leqq \gamma\left(1-\frac{\eta}{2}+\frac{\eta}{2}\right)=\gamma, \quad i \neq j_{1},
$$

whereas for $j_{1}$

$$
\left\langle k_{j_{1}}^{(n)}(\xi+\bar{\xi}+t)\right\rangle \geqq\left|g\left(k_{j_{1}}^{(n)}(\xi+\bar{\xi})\right)\right|-\left\langle k_{j_{1}}^{(n)} t\right\rangle>\gamma .
$$

Thus, for sufficiently large $n$,

$$
\begin{array}{r}
B\left(k^{(n)}\left(r, j_{1}\right)\right) \geqq \mu\left\{v: v=\xi+\xi+t, 0 \leqq t \leqq 1,\left\langle k_{i}^{(n)} t\right\rangle\left\langle\gamma \frac{\eta}{2}, i=1, \cdots, r\right\}\right. \\
=P\left\{\left\langle k_{i}^{(n)} x\right\rangle\left\langle\gamma \frac{\eta}{2}, i=1, \cdots, r\right\}=A_{r \eta / 2}\left(k^{(n)}(r)\right) \geqq C_{6}^{-1}\left(r, \frac{\gamma \eta}{2}\right) .\right.
\end{array}
$$

This contradicts (3.34) and therefore proves the lemma.

LEMma 6. If $p \rightarrow \infty, m \rightarrow \infty$ such that $m(2 \gamma)^{p} \rightarrow \lambda$ then for some $C_{9}$, depending on $\gamma, r, \lambda$ only, and $j \leqq p$

$$
\begin{aligned}
\exp \left(-C_{9}(2 \gamma)^{(p-j) / 2}(1\right. & +p-j)) \\
& \leqq \frac{1}{m-r+1} \sum_{\substack{k \neq k_{1}, \cdots, k_{r-1} \\
1 \leq k \leq m}}\left(\frac{A_{\gamma}\left(k_{1}, \cdots, k_{r-1}, k\right)}{A_{\gamma}\left(k_{1}, \cdots, k_{r-1}\right) 2 \gamma}\right)^{j} \\
& \leqq \exp \left(C_{9}(2 \gamma)^{(p-j) / 2}(1+p-j)\right) .
\end{aligned}
$$

Proof. Put

(3.46) $\nu^{(j)}(\gamma, k(r-1))=\frac{1}{m-r+1} \sum_{\substack{k \neq k_{1}, \cdots, k_{r-1} \\ 1 \leq k \leq m}}\left(\frac{A_{\gamma}\left(k_{1}, \cdots, k_{r-1}, k\right)}{A_{\gamma}\left(k_{1}, \cdots, k_{r-1}\right) 2 \gamma}\right)^{j}$.

In this proof we assume $k(r-1)$ and $\gamma$ fixed and shall abbreviate $\nu^{(j)}(\gamma, k(r-1))$ by $\nu^{(j)}$. For the same fixed $k(r-1)$ and $\gamma$ we put

$$
N^{\prime}(j)=\sum_{\substack{k \neq k_{1}, \cdots, k_{r} \\ 1 \leq k \leq m}} Y_{k}^{j}(\gamma)
$$

Then (cf. (3.32)),

$$
\nu^{(j)}=\frac{E\left(N^{\prime}(j) \mid Y_{k_{1}}^{j}=\cdots=Y_{k_{r-1}}^{j}=1\right)}{(m-r+1) \cdot(2 \gamma)^{j}} .
$$

The proof will very much resemble the proof of Lemma 3. 


$$
\begin{aligned}
& E\left(N^{\prime}(j) \mid Y_{k_{1}}^{j}=\cdots=Y_{k_{r-1}}^{j}=1\right) \\
& (3.47)=\sum_{\substack{1 \leq t_{1}<\cdots<t_{s} \leq m \\
t_{u} \neq k_{v}}} P\left\{F\left(k_{1}, \cdots, k_{r-1}, t_{1}, \cdots, t_{s} ; j-1\right) \mid Y_{k_{1}}^{j-1}=\cdots\right. \\
& \left.\quad=Y_{k_{r-1}}^{j-1}=1\right\} \\
& \cdot E\left(N^{\prime}(j) \mid Y_{k_{1}}^{j}=\cdots=Y_{k_{r-1}}^{j}=1, F\left(k_{1}, \cdots, k_{r-1}, t_{1}, \cdots, t_{s} ; j-1\right)\right) .
\end{aligned}
$$

However,

$$
E\left(N^{\prime}(j) \mid F\left(k_{1}, \cdots, k_{r-1}, t_{1}, \cdots, t_{s} ; j-1\right)\right)=2 \gamma s
$$

and thus by Lemma 1 (compare (3.23), (3.24)),

$$
E\left(\left(N^{\prime}(j)-2 \gamma s\right)^{2} \mid F\left(k_{1}, \cdots, k_{r-1}, t_{1}, \cdots, t_{s} ; j-1\right)\right) \leqq \frac{s}{2}\left(C_{1}+(\log s)^{2}\right) .
$$

Hence, by Lemma 4

$$
\begin{aligned}
& E\left(\left(N^{\prime}(j)-2 \gamma s\right)^{2} \mid Y_{k_{1}}^{(j)}=\cdots=Y_{k_{r-1}}^{(j)}=1,\right. \\
& \leqq \frac{s\left(C_{1}+(\log s)^{2}\right)}{2 P\left\{Y_{k_{1}}^{(j)}=\cdots=Y_{k_{r-1}}^{(j)}=1 \mid F\left(k_{1}, \cdots, k_{r-1}, t_{1}, \cdots, t_{s} ; j-1\right)\right.} \\
& =\frac{s\left(C_{1}+(\log s)^{2}\right)}{2 A\left(k_{1}, \cdots, k_{r-1}\right)} \leqq C_{6}(r-1, \gamma) \frac{s}{2}\left(C_{1}+(\log s)^{2}\right) .
\end{aligned}
$$

By (3.12) and (3.48) one has

$$
\begin{aligned}
E\left(N^{\prime}(j) \mid Y_{k_{1}}^{j}=\cdots=Y_{k_{r-1}}^{j}\right. & =1, \\
& \left.\left.F\left(k_{1}, \cdots, k_{r-1}, t_{1}, \cdots, t_{s} ; j-1\right)\right)-2 \gamma s\right) \\
& \leqq C_{10}(r, \gamma) s^{1 / 2}(1+\log s)
\end{aligned}
$$

for appropriate $C_{10}$.

Since, under the condition $F\left(k_{1}, \cdots, k_{r-1}, t_{1}, \cdots, t_{s} ; j-1\right)$

$$
N^{\prime}(j-1)=s,
$$

(3.47) and (3.49) give immediately

$$
\begin{aligned}
& \mid E\left(N^{\prime}(j) \mid Y_{k_{1}}^{j}=\cdots\right. \\
& \left.\quad=Y_{k_{r-1}}^{j}=1\right)-2 \gamma E\left(N^{\prime}(j-1) \mid Y_{k_{1}}^{j-1}=\cdots=Y_{k_{r-1}}^{j-1}=1\right) \mid \\
& \quad \leqq C_{10} E\left(N^{\prime 1 / 2}(j-1)\left(1+\log N^{\prime}(j-1)\right) \mid Y_{k_{1}}^{j-1}=\cdots=Y_{k_{r-1}}^{j-1}=1\right) \\
& \quad \leqq 5 C_{10} E\left(N^{\prime 2 / 4}(j-1) \mid Y_{k_{1}}^{j-1}=\cdots=Y_{k_{r-1}}^{j-1}=1\right) .
\end{aligned}
$$

Thus 


$$
\nu^{(j)} \leqq \nu^{(j-1)}+\frac{5 C_{10}\left(\nu^{(j-1)}\right)^{3 / 4}}{(m-r+1)^{1 / 4}(2 \gamma)^{j / 4+1}}, \quad \quad \nu^{(0)}=1 .
$$

First we get from (3.51) by induction,

$$
\nu^{(j)} \leqq \exp \left(5 C_{10} \sum_{u=0}^{j-1}(m-r+1)^{-1 / 4}(2 \gamma)^{-u / 4-1}\right)
$$

which is bounded for $j \leqq p$. Then we complete the proof from (3.50) as in Lemma 3 by applying Hölder's and Jensen's inequalities and induction on $j\left({ }^{(}\right)$.

An immediate corollary of (3.45) is

$$
\begin{aligned}
\frac{1}{m-r+1} \sum_{\substack{k \neq k_{1}, \cdots, k_{r-1} \\
1 \leq k \leq m}}\left(\frac{A_{\gamma}\left(k_{1}, \cdots, k_{r-1}, k\right)}{A_{\gamma}\left(k_{1}, \cdots, k_{r-1}\right) 2 \gamma}\right)^{3[p / 3]+3} & \leqq(2 \gamma)^{-3} \exp C_{9}=C_{11} \text {, say. }
\end{aligned}
$$

We are now in the position to prove (3.5). Put

$$
U_{r}(k(r))=\left(\frac{A(k(r))}{(2 \gamma)^{r}}\right)^{[p / 3]+1} .
$$

If we consider $U_{r}$ as a random variable taking each of the values $U_{r}(k(r))$ with probability $1 / m(m-1) \cdots(m-r+1)$ then (3.6) and Lemma 3 state

$$
\begin{aligned}
E U_{r} & =\frac{1}{m(m-1) \cdots(m-r+1)} \sum_{k(r)}\left(\frac{A(k(r))}{(2 \gamma)^{r}}\right)^{[p / 3]+1} \\
& \geqq \exp \left(-C_{4}(2 \gamma)^{(2 p-3) / 3} p^{2}\right) \quad \text { for sufficiently large } p .
\end{aligned}
$$

Similarly

$$
E U_{r}^{2} \leqq \exp C_{4}(2 \gamma)^{(p-6) / 3} p^{2}
$$

and consequently

$$
\operatorname{var}\left(U_{r}\right)=E U_{r}^{2}-\left(E U_{r}\right)^{2} \leqq C_{12}(\gamma, r, \lambda) p^{2}(2 \gamma)^{p / 3}
$$

for appropriate $C_{12}$. In other words

$$
U_{r} \rightarrow 1 \text { in probability }(p \rightarrow \infty)
$$

and

$$
\frac{1}{m(m-1) \cdots(m-r+1)} \sum_{k(r)}\left(\frac{A(k(r))}{(2 \gamma)^{r}}\right)^{p}=E U_{r}^{p /([p / 3]+1)}
$$

will indeed tend to one if we can show

$$
\lim \frac{1}{m(m-1) \cdots(m-r+1)} \sum_{k(r): U^{8} r \geq i^{r}} U_{r}^{3}(k(r))=0
$$


$[9$, p. 184 B].

Unfortunately, Lemma 3 alone does not seem to be strong enough to prove (3.53), and we have to proceed by induction. (3.53) certainly holds for $r=1$ and let us assume it has already been proved with $r$ replaced by $r-1$. We shall then prove that it also holds for $r$. For this purpose, we define

$$
V_{r}(k(r))=\left\{\begin{array}{l}
\left(\frac{A_{\gamma}\left(k_{1}, \cdots, k_{r}\right)}{A_{\gamma}\left(k_{1}, \cdots, k_{r-1}\right) 2 \gamma}\right)^{[p / 3]+1} \text { if } \frac{A_{\gamma}\left(k_{1}, \cdots, k_{r}\right)}{A_{\gamma}\left(k_{1}, \cdots, k_{r-1}\right)} \leqq C_{13}(\gamma, r), \\
\frac{1}{m-r+1} \sum_{k \neq k_{1}, \cdots, k_{r-1}}\left(\frac{A_{\gamma}\left(k_{1}, \cdots, k_{r-1}, k\right)}{A_{\gamma}\left(k_{1}, \cdots, k_{r-1}\right) 2 \gamma}\right)^{[p / 3]+1} \text { otherwise }
\end{array}\right.
$$

where

$$
C_{13}(\gamma, r)=\max \left(1-C_{7}(\gamma, r),(2 \gamma)\right)<1
$$

Any set of $r$ different integers $k_{1}, \cdots, k_{r} \leqq m$ will appear in $r$ ! orders as a $k(r)$. For some $j$

$$
\frac{A_{\gamma}(k(r))}{A_{\gamma}(k(r, j))} \leqq C_{13}(\gamma, r)
$$

by Lemma 5 .

This $j$ will appear in $(r-1)$ ! permutations of $1, \cdots, r$ at the end. Thus taking into account

$$
\begin{aligned}
& \left(\frac{A(k(r))}{(2 \gamma)^{r}}\right)^{3[p / 3]+3}=\left(\frac{A(k(r-1))}{(2 \gamma)^{r-1}}\right)^{3[p / 3]+3}\left(\frac{A(k(r))}{A(k(r-1)) 2 \gamma}\right)^{3[p / 3]+3},
\end{aligned}
$$

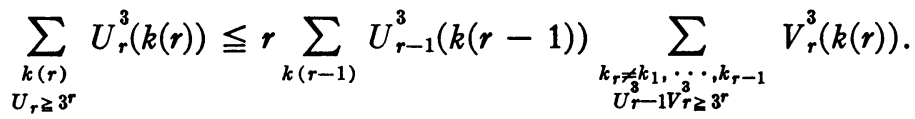

Since $U^{3} V^{3} \geqq 3^{r}$ implies $U^{3} \geqq 3^{r-1}$ or $V \geqq 3$ one obtains from (3.56)

$$
\begin{aligned}
& \frac{1}{m(m-1) \cdots(m-r+1)} \sum_{\substack{k(r) \\
U_{r} \geq 3^{r}}} U_{r}^{3}(k(r)) \\
& \leqq \frac{r}{m(m-1) \cdots(m-r+2)} \sum_{\substack{k(r) \\
U_{r-1} 3^{r-1}}} U_{r-1}^{3}(k(r-1)) \frac{1}{m-r+1} \\
& \cdot \sum_{k_{r} \neq k_{1}, \cdots, k_{r-1}} V_{r}^{3}(k(r)) \\
& +\frac{r}{m(m-1) \cdots(m-r+2)} \sum_{\substack{k(r-1) \\
m(n-1)}} U_{r-1}^{3}(k(r-1)) \frac{1}{m-r+1} \\
& \quad \cdot \sum_{\substack{k_{r \neq 1} k_{1}, \cdots, k_{r-1} \\
V_{r \geq 3}}} V_{r}^{3}(k(r)) .
\end{aligned}
$$


By the induction hypothesis and (3.52) the first sum in the right-hand side of (3.57) tends to zero. As for the second term, we have

$$
\begin{aligned}
& \frac{1}{m(m-1) \cdots(m-r+2)} \sum_{k(r-1)} U_{r-1}^{2}(k(r-1)) \frac{1}{m-r+1} \\
& \cdot \sum_{k \neq k_{1}, \cdots, k_{r-1}}\left(\frac{A\left(k_{1}, \cdots, k_{r-1}, k\right)}{A(k(r-1)) 2 \gamma}\right)^{2[p / 3]+2} \leqq \exp \left(C_{4}(2 \gamma)^{(p-6) / 3} p^{2}\right.
\end{aligned}
$$

by (3.6) and Lemma 3. On the other hand by (3.6), Lemma 3 and Lemma 6

$$
\begin{gathered}
\frac{1}{m(m-1) \cdots(m-r+2)} \sum_{k(r-1)} U_{r-1}^{2}(k(r-1)) \\
\cdot\left(\frac{1}{m-r+1} \sum_{k \neq k_{1}, \cdots, k_{r-1}}\left(\frac{A\left(k_{1}, \cdots, k_{r-1}, k\right)}{A(k(r-1)) 2 \gamma}\right)^{[p / 3]+1}\right)^{2} \\
\geqq \exp \left(-C_{4}(2 \gamma)^{(p-6) / 3} p^{2}\right) \exp \left(-2 C_{9}(2 \gamma)^{(p-3) / 3} p\right) .
\end{gathered}
$$

Putting

$$
D(k(r-1))=\frac{1}{m-r+1} \sum_{\substack{k \neq k_{1}, \cdots, k_{r-1} \\ 1 \leq k \leq m}}\left(\frac{A\left(k_{1}, \cdots, k_{r-1}, k\right)}{A(k(r-1)) 2 \gamma}\right)^{[p / 3]+1}
$$

and subtracting (3.59) from (3.58) one sees

$$
\begin{gathered}
\frac{1}{m(m-1) \cdots(m-r+2)} \sum_{k(r-1)} U_{r-1}^{2}(k(r-1)) \\
(3.60) \cdot \frac{1}{m-r+1} \sum_{k \neq k_{1}, \cdots, k_{r-1}}\left(\left(\frac{A\left(k_{1}, \cdots, k_{r-1}, k\right)}{A(k(r-1)) 2 \gamma}\right)^{[p / 3]+1}-D(k(r-1))\right)^{2} \\
\leqq C_{14}(\gamma, r, \lambda)(2 \gamma)^{p / 3} p^{2}
\end{gathered}
$$

for appropriate $C_{14}$. Thus

$$
\begin{aligned}
\frac{1}{m-r+1} \sum_{k \neq k_{1}, \cdots, k_{r-1}}\left(\left(\frac{A\left(k_{1}, \cdots, k_{r-1}, k\right)}{A(k(r-1)) 2 \gamma}\right)^{[p / 3]+1}\right. & -D(k(r-1)))^{2} \\
& \leqq C_{14}(2 \gamma)^{p / 3} p^{2} C_{13}^{-p / 0}
\end{aligned}
$$

except for a set $S$ of $(r-1)$-tuples $k(r-1)$ for which

$$
\frac{1}{m(m-1) \cdots(m-r+2)} \sum_{k(r-1) \in S} U_{r-1}^{2}(k(r-1)) \leqq C_{13}^{p / 6} .
$$

However, by (3.52) 


$$
\begin{array}{r}
\frac{1}{m(m-1) \cdots(m-r+2)} \sum_{k(r-1) \in S} U_{r-1}^{3}(k(r-1)) \frac{1}{m-r+1} \sum_{k \neq k_{1}, \cdots, k_{r-1}} V_{r}^{3}(k(r)) \\
\leqq \frac{2 C_{11}}{m(m-1) \cdots(m-r+2)} \sum_{k(r-1) \in S} U_{r-1}^{3}(k(r-1)) \\
\leqq \frac{2 C_{11}}{m(m-1) \cdots(m-r+2)} \sum_{U_{r-1} \geq 3^{r-1}} U_{r-1}^{3}(k(r-1)) \\
+\frac{2 C_{11} \cdot 3^{r-1}}{m(m-1) \cdots(m-r+2)} \sum_{k(r-1) \in S} U_{r-1}^{2}(k(r-1))
\end{array}
$$

Both these terms tend to zero, the first by the induction hypothesis and the second by (3.62) and (3.55). In view of (3.57) it remains to prove

$$
\begin{aligned}
\frac{1}{m(m-1) \cdots(m-r+2)} \sum_{k(r-1) \notin S} U_{r-1}^{3}(k(r-1)) & \frac{1}{m-r+1} \sum_{\substack{k_{r} \neq k_{1}, \cdots, k_{r-1} \\
V_{r} \geq 3}} V_{r}^{3}(k(r)) \rightarrow 0 .
\end{aligned}
$$

Let $k(r-1) \notin S$ be fixed and look at $V_{r}$ as a random variable which takes each of the values $V_{r}(k(r))$ with probability $1 /(m-r+1)$. Then

$$
\begin{aligned}
& E\left(V_{r}-D_{r}(k(r-1))\right)^{2} \\
& \quad \leqq \frac{1}{m-r+1} \sum_{k \neq k_{1}, \cdots, k_{r-1}}\left(\left(\frac{A\left(k_{1}, \cdots, k_{r-1}, k\right)}{A(k(r-1)) 2 \gamma}\right)^{[p / 3]+1}-D(k(r-1))\right)^{2} \\
& \quad \leqq C_{14}(2 \gamma)^{p / 3} p^{2} C_{13}^{-p / 6} \text { by }(3.61) .
\end{aligned}
$$

On the other hand

$$
0 \leqq V_{r} \leqq \max \left(\left(\frac{C_{13}}{2 \gamma}\right)^{[p / 3]+1}, \quad D(k(r-1))\right)
$$

while $D(k(r-1)) \leqq 3 / 2$ for sufficiently large $p$ by Lemma 6 . Thus, by Lemma 2, (3.13)

$$
\begin{aligned}
& \frac{1}{m-r+1} \sum_{\substack{k \neq k_{1}, \cdots, k_{r} \\
V_{r} \geq 3}} V_{r}^{3}(k(r)) \\
& \leqq C_{2}(3)\left(\frac{3}{2}+\left(\frac{C_{13}}{2 \gamma}\right)^{[p / 3]+1}\right) \cdot C_{14}(2 \gamma)^{p / 3} p^{2} C_{13}^{-p / 6} \rightarrow 0 \quad(p \rightarrow \infty) .
\end{aligned}
$$


Since, by Lemma 3

$$
\frac{1}{m(m-1) \cdots(m-r+2)} \sum_{k(r-1)} U_{r-1}^{3}(k(r-1))
$$

is uniformly bounded, (3.63) follows from (3.64) and this implies (3.53). By induction (3.53) and (3.5) follow now for all $r$ and this proves the theorem.

Corollary. If $p \rightarrow \infty, m \rightarrow \infty$ such that

$$
m(2 \gamma)^{p} \rightarrow \infty \quad \text { sufficiently slow, }
$$

then

$$
P\left\{\frac{N(m, \gamma, p)-m(2 \gamma)^{p}}{\left(m(2 \gamma)^{p}\right)^{1 / 2}} \leqq \alpha\right\} \rightarrow \frac{1}{(2 \pi)^{1 / 2}} \int_{-\infty}^{\alpha} e^{-t^{2} / 2} d t
$$

Proof. This follows from Theorem 3, since for random variables $X(\lambda)$, having a Poisson distribution with mean $\lambda$, one has $[6$, p. 116, Ex. 4.9]

$$
\lim _{\lambda \rightarrow \infty} P\left\{\frac{X(\lambda)-\lambda}{\lambda^{1 / 2}} \leqq \alpha\right\}=\frac{1}{(2 \pi)^{1 / 2}} \int_{-\infty}^{\alpha} e^{-t^{2} / 2} d t
$$

Our estimates are not sharp enough, however, to tell how fast $m(2 \gamma)^{p}$ may tend to infinity.

One might want to prove Theorem 3 for any interval of length $2 \gamma$. More precisely, put

$$
Y_{k}^{j}(\delta, 2 \gamma+\delta)= \begin{cases}1 & \text { if there exist integers } n_{1}, \cdots, n_{j} \text { such that } \\ 0 & \delta+n_{i} \leqq k x_{i} \leqq \delta+2 \gamma+n_{i}, i=1, \cdots, j,\end{cases}
$$

As long as

$$
-\frac{1}{2}<\delta<0<\delta+2 \gamma<\frac{1}{2}
$$

one can still follow the proof of Theorem 3. In fact, the only places in the above proof where a difference between $Y(\gamma)$ and $Y(\delta, 2 \gamma+\delta)$ might come in are the Lemmas 1, 4 and 5. However, the estimate (3.8) remains valid (cf. [8]) so that the proof of Lemma 1 needs no change and one easily sees also that Lemmas 4 and 5 remain valid. This is not so when (3.65) is violated. The analogue of Lemma 4 is then false for certain choices of $\boldsymbol{\gamma}$ and $\delta$. We formulate this with another generalization in

THEOREM 4. If $\gamma, \delta$ satisfy (3.65) and if $p \rightarrow \infty, m \rightarrow \infty$ such that $m(2 \gamma)^{p} \rightarrow \lambda$ and if for every $m, k_{1}(m), \cdots, k_{m}(m)$ are $m$ different positive integers, then

$$
\lim P\left\{\sum_{i=1}^{m} Y_{k_{i}(m)}^{p}(\delta, 2 \gamma+\delta)=k\right\}=e^{-\lambda} \frac{\lambda^{k}}{k !}, \quad k=0,1,2, \cdots .
$$


The transition to arbitrary sequences $\left\{k_{i}(m), i=1, \cdots, m\right\}$ of integers requires no change of proof since our estimates are uniform in $\left\{k_{i}(m)\right\}$. Finally we mention the possibility of taking intervals of length $2 \gamma$ at a random location. I.e. let $\delta_{1}, \delta_{2}, \cdots$ also be random variables, independent of each other and of $x_{1}, x_{2}, \cdots$, and each with a uniform distribution on $[0,1]$. Put

$$
\tilde{Y}_{k}^{j}(\gamma)=\left\{\begin{array}{l}
1 \text { if there exist integers } n_{1}, \cdots, n_{j} \text { such that } \\
\qquad \delta_{i}+n_{i} \leqq k x_{i} \leqq \delta_{i}+2 \gamma+n_{i}, i=1, \cdots, j, \\
0 \text { otherwise. }
\end{array}\right.
$$

Then Theorem 4 holds with $Y(\delta, 2 \gamma+\delta)$ replaced by $\tilde{Y}(\gamma)$. Again the proof is almost identical with that of Theorem 3 .

\section{REFERENCES}

1. P. Erdös and A. Rényi, $A$ probabilistic approach to problems of diophantine approximation, Illinois J. Math. 1 (1957), 303-315.

2. P. Erdös, P. Szüsz and P. Turan, Remarks on the theory of diophantine approximations, Colloq. Math. 6 (1958), 119-126.

3. Bernard Friedman and Ivan Niven, The average first recurrence time, Trans. Amer. Math. Soc. 92 (1959), 25-34.

4. G. H. Hardy, J. E. Littlewood and G. P6lya, Inequalities, 2d ed., Cambridge Univ. Press, Cambridge, 1952.

5. G. H. Hardy and E. M. Wright, An introduction to the theory of numbers, 3d ed., Oxford Univ. Press, London, 1954.

6. Maurice G. Kendall and Alan Stuart, The advanced theory of statistics, Vol. I, Hafner, New York, 1958.

7. Harry Kesten, Uniform distribution mod 1, Ann. of Math. 71 (1960), 445-471.

8. W. J. LeVeque, On the frequency of small fractional parts in certain real sequences. III, J. Reine Angew. Math. 202 (1959), 215-220.

9. Michel Loève, Probability theory, Van Nostrand, New York, 1955.

10. E. C. Titchmarsh, The theory of functions, corrected 2d ed., Oxford Univ. Press, London, 1952.

The Hebrew University, JERUSALEM, ISRAEL 